\title{
Comparative Study of Active Release Technique and Myofascial Release Technique in Treatment of Patients with Non-Specific Low Back Pain
}

\section{Hamza M Shaheen ${ }^{1 *}$, Karim Ghuiba ${ }^{2}$, Alaa Abu Sakour ${ }^{1}$, Ameer Tayseer $^{1}$ and Sayel Abu Daowd ${ }^{1}$}

${ }^{1}$ Department of Physiotherapy, Faculty of Allied Medical Sciences, Palestine Ahlyia

University, Bethlehem, Palestine

${ }^{2}$ Department of Physical Therapy for Musculoskeletal Disorders and its Surgery,

Faculty of Physical Therapy, CarioUniversity, Egypt

*Corresponding Author: Hamza M Shaheen, Department of Physiotherapy, Faculty of Allied Medical Sciences, Palestine Ahlyia University, Bethlehem, Palestine.
Received: June 24, 2021

Published: July 12, 2021

(C) All rights are reserved by Hamza M

Shaheen., et al.

\section{Abstract}

Aim: To compare between the efficacy of Active release technique and Myofascial Release Technique on pain and range of motion in patients with non-specific low back pain.

Subjects and Methods: 20 patients with low back pain who were randomly assigned to one of two groups that received Active release technique ( $n=10$; mean age, 33.3 years) or Myofascial Release Technique ( $n=10$; mean age, 28.4 years). Both groups were received their programs for one week; 7 sessions per week.

Main Measures: VAS, ROM, and functional disability. An assessment was made for each group at the initial visit (before initiation of treatment) and at end of the program.

Results: There was a significant difference between group (A) and (B) in pain level where P-value was $(<0.005)$, and there was a significant difference between group (A) and (B) in ROM where the P-value was $(<0.005)$ and there was a significant difference between group (A) and (B) in LBDI where the P-value was $(<0.005)$.

Conclusion: There was a significant difference between active release technique and myofascial release technique as regard to pain reduction, improvement ROM and functional disability. Which active release technique allows a greater degree of pain relief and allows a greater improvement in ROM and function disability than the myofascial release technique for patients with low back pain.

Keywords: Low Back Pain; Active Release Technique; Myofascial Release Technique

\section{Introduction}

Low back pain (LBP) incidence was estimated at between 4 percent -56 percent of the general population per year [1]. Between 60 and 80 per cent of the population will experience LBP during their lifetime and become up to 15 per cent. LBP is second only to the common cold that affects as many as 20 per cent of the population annually in missing workdays in the United States [2].
Estimates of annual prevalence of LBP range from 41 to 65 per cent. And healthcare economists estimate that up to 80 percent of healthcare costs associated with LBP are produced by 15 percent of cases [3].

LBP is a major source of incapacity and economic costs. Most LBP appears to be of unknown etiology, despite the prevalence and social costs. Reasons for this assertion include a lack of responsive- 
ness to special tests used to determine LBP, a high incidence to anatomical abnormalities observed on diagnostic imaging, a failure to demonstrate a strong correlation between anatomical abnormality and clinical symptomatology, and a failure of clinical evaluation to predict symptoms and impairment levels [4].

Lumbar spine muscles have been shown to act as primary sources of back pain and buttock pain. Disorders that predominantly affect the lumbar spine musculature include injury to muscle strain, spasm or guarding, and myofascial complaints, such as trigger point. It has found that muscle activation patterns of individuals with LBP differ from those of ordinary people [5].

Perhaps the most common of soft tissue therapy/mobilization techniques used by manual therapists is the active release technique (ART). ART is a proprietary, non-invasive treatment of soft tissue that locates and breaks down the cicatric tissue and adhesions that cause discomfort, stiffness, fatigue, numbness and physical dysfunction [6].

Myofascial release technique (MFR) is used to treat patients with low back pain but few formal reports of its success rate are available. MFR is the application of a low load, long-lasting stretch to the myofascial complex with the goal of restoring optimum duration, reducing pain and improving function [7].

\section{Materials and Methods}

Twenty male and female patients with age from 20 - 50 years suffering from low back pain participated in this study. All patients were randomly selected from the orthopedic surgeon. Patients were randomly assigned into 2 groups A, B. All patients of the study were informed about the study steps, and the expected benefits were explained before signing the informed consent. All patients included in this study were to sign informed consent:

1. Group $A(n=10)$ active release technique was applied for 7 sessions for one week.

2. Group B ( $\mathrm{n}=10)$ myofascial Release Technique was applied for 7 sessions for one week.

Inclusion criteria:

- Patients with lower back pain.

- Age ranged from 20 to 50 years.

- The patient had a 3-month or longer history of low back pain.
Exclusion criteria:

- History of Sciatica or any radiating pain,

- Back deformities,

- History of trauma or fracture or surgery in the lower back,

- And any skin diseases in the low back area.

Treatment procedures:

- Active release technique: The patient was in a sitting position on the treatment table with his hips flexed and knees flexed and hanging off the end of the table. The back of the patient then extended to shorten the extensor group. The therapist sitting behind the patient then evaluated the texture and tightness of the extensor group of the back by palpating and manually contacting the exterior skin to see where maximum tightness could be felt. The therapist then placed tension on the bellies of the extensor group of the back longitudinally at a specific tension and asked the patient to flex his back as per ART protocol. This cycle was repeated 5 more times on each patient. The treatment was given for 7 sessions.

- Myofascial release technique: The patient was in a sitting position on the treatment table with his hips flexed and knees flexed and hanging off the end of the table. The back of the patient was then flexed to lengthen the extensor group. The therapist sitting behind the patient then evaluated the texture and enlargement of the extensor group of the back by palpating and manually contacting the exterior skin to see where maximum enlargement could be felt. The therapist then placed tension on the bellies of the extensor group of the back longitudinally at a specific tension and asked the patient to flex his back as per MRT protocol. This cycle was repeated 5 more times on each patient. The treatment was given for 7 sessions.

Evaluation parameters:

- Visual analog scale: It is a graphical rating scale, which is commonly interpreted as a valid pain intensity report and has been used to record pain intensity levels. VAS is a-10 cms line, was written at one end (no pain = zero) and was written at the other end (the patient has ever felt the worst pain = 10 ). Each subject was asked to mark and score on the line at the point representing its pain intensity [8]. 
- Tape measurement: One of the simplest methods for measuring range of motion and length of the muscle is tape measure (or ruler) tape measurements can be made from cloth or metal. They may possess a scale of centimetres, an inch, or both. The tape measurement is easy to use and ready to use in most clinics [9].

- Oswestry low back pain disability questionnaire: The Oswestry Disability Index (also known as the Oswestry Low Back Pain Disability Questionnaire) is an extremely useful instrument used by researchers and evaluators of the condition to assess the permanent functional impairment of an individual. The test is considered the 'gold standard' of functional outcome tools with low back [10].

\section{Results}

The purpose of this study was to investigate the difference between the effect of active release technique and myofascial release technique on pain, range of motion and functional disability in patients with low back pain.

\section{General characteristics of the subjects}

In this study, 20 patients with low back pain were assigned randomly into two groups 10 patients in each group.

Group (A): Ten patients were included in this group who received active release therapy (ART). The data in table 1 and figure 1 represents the mean and standard deviation of their age $(33.20 \pm$ 11.053 ) years, weight (78.5 \pm 7.93 ) kilograms $(\mathrm{Kg})$, height $(176.30$ $\pm 7.424)$ centimeters $(\mathrm{cm})$, and BMI $(25.18 \pm 0.74) \mathrm{Kg} / \mathrm{m}^{2}$.

Group (B): Ten patients were included in this group that received myofascial release technique (MRT). The data in table 1 and figure 1 represents the mean and standard deviation of their age $(28.30 \pm 7.675)$ years, weight $(75.3 \pm 5.41)$ kilograms $(\mathrm{Kg})$, height $(173.70 \pm 4.620)$ centimeters $(\mathrm{cm})$ and BMI $\left(\mathrm{Kg} / \mathrm{m}^{2}\right)$.

There was no significant difference between both groups in their ages, weights, heights, and BMI (P-Value < 0.05).

\section{Within subjects}

For the analysis of each dependent variable, the univariate test was conducted using a Bonferroni adjusted alpha level of 0.025 $(0.05 / 2)$ as shown in table 2 and 3.

\begin{tabular}{|l|c|c|c|}
\hline \multicolumn{1}{|c|}{ Variables } & ART group & MRT group & P-value \\
\hline & $\overline{\mathbf{x}} \pm \mathbf{s}$ & $\overline{\mathbf{x}} \pm \mathbf{~ s}$ & \\
\hline Age & $33.20 \pm 11.053$ & $28.30 \pm 7.675$ & 0.265 \\
\hline Weight $(\mathrm{kg})$ & $78.5 \pm 7.93$ & $75.3 \pm 5.41$ & 0.022 \\
\hline Height $(\mathrm{cm})$ & $176.30 \pm 7.424$ & $173.70 \pm 4.620$ & 0.36 \\
\hline BMI $\left(\mathrm{kg} / \mathrm{m}^{2}\right)$ & $25.18 \pm 0.74$ & $25.32 \pm 1.01$ & 0.003 \\
\hline
\end{tabular}

Table 1: General characteristics of patients in both groups (A\&B). *SD: Standard Deviation, P: Probability.

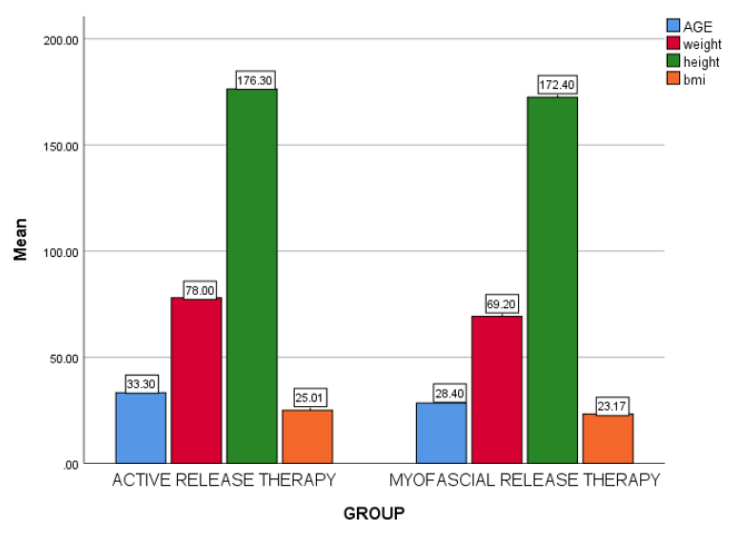

Figure 1: Mean and \pm SD of the age, weight, height and BMI for groups (A, B).

For group (A) there was a significant difference between pre and post treatment pain level as the pain level pre-treatment was $(6.40 \pm 0.966)$ and for post-treatment was (1.20 \pm 0.632$)$ (P-value $<0.005$ ) and there was a significant difference between pre and post treatment for ROM as ROM pre-treatment was flexion (5.20 $\pm 0.789)$, extension $(1.90 \pm 0.738)$ lateral bending RT $(9.5 \pm 2.12)$, lateral bending LT $(9.5 \pm 2.12)$, rotation RT ( $4.7 \pm 0.67)$ and rotation LT $(5.00 \pm 0.66)$ and for post-treatment was flexion ( $9.20 \pm 0.632)$, extension ( $4.6 \pm 0.699$ ) lateral bending RT $(14.4 \pm 2.06)$, lateral bending LT $(14.4 \pm 2.06)$, rotation RT $(8.1 \pm 0.56)$ and rotation LT $(8.2 \pm 0.78)(\mathrm{P}$-value $<0.05)$, and there was a significant difference between pre and post treatment for LBPI as pre-treatment was $(31.860 \pm 12.2448)$ and for post-treatment was (14.660 \pm 4.8983$)$ (P-value $<0.05$ ), as shown in table 2. 


\begin{tabular}{|l|c|c|c|}
\hline \multirow{2}{*}{} & \multicolumn{2}{|c|}{ ART group } & \multirow{2}{*}{ p-value } \\
\cline { 2 - 3 } & \multicolumn{2}{|c|}{$\overline{\mathbf{x}} \pm \mathbf{~}$} & \\
\cline { 2 - 3 } & $6.40 \pm 0.966$ & $1.20 \pm 0.632$ & 0.004 \\
\hline VAS & $5.20 \pm 0.789$ & $9.20 \pm 0.632$ & 0.005 \\
\hline Flexion & $1.90 \pm 0.738$ & $4.6 \pm 0.699$ & 0.004 \\
\hline Extension & $9.5 \pm 2.12$ & $14.4 \pm 2.06$ & $<0.005$ \\
\hline (right) & $9.5 \pm 2.12$ & $14.4 \pm 2.06$ & $<0.005$ \\
\hline Lateral flexion (left) & $4.7 \pm 0.67$ & $8.1 \pm 0.56$ & $<0.005$ \\
\hline Rotation (right) & $5.00 \pm 0.66$ & $8.2 \pm 0.78$ & $<0.005$ \\
\hline Rotation (left) & $31.860 \pm$ & $14.660 \pm$ & \multirow{2}{*}{0.005} \\
\hline LBP index & 12.2448 & 4.8983 & \\
\hline
\end{tabular}

Table 2: Mean and \pm SD, and univariate test between pre and post treatment of the dependent variables in group (A).

*SD: Standard Deviation, P: Probability.

For group (B) there was a significant difference between pre and post treatment pain level as the pain level pre-treatment was $(6.30 \pm 0.823)$ and for post-treatment was (3.20 \pm 0.789$)$ (P-value $<0.05)$, and there was a significant difference between pre and post treatment for ROM as ROM pre-treatment was flexion (6.30 \pm $0.675)$, extension (1.80 \pm 0.789 ) lateral bending RT (10.1 \pm 1.91$)$, lateral bending LT $(9.90 \pm 1.52)$, rotation RT $(4.7 \pm 0.67)$ and rotation LT $(5.00 \pm 0.66)$ and for post-treatment was flexion (8.90 \pm 1.197), extension (3.80 \pm 0.919 ) lateral bending RT (13.7 \pm 1.56$)$, lateral bending LT $(13.7 \pm 1.56)$, rotation RT $(7.1 \pm 0.56)$ and rotation LT $(7.2 \pm 0.78)$ (P-value $<0.05)$ and there was a significant difference between pre and post treatment for LBPI as pre-treatment was (31.690 \pm 13.1575$)$ and for post-treatment was (19.460 \pm 6.7421) (P-value was < 0.05), as shown in table 3.

\section{Between groups}

For the analysis of each dependent variable the univariate test was conducted using a Bonferroni adjusted alpha level of 0.025 $(0.05 / 2)$.

Pre treatment there was no significant difference between group (A) and (B) in pain level where P-value more than 0.05 , and there was no significant difference between group (A) and (B) in ROM where P-value was (>0.05) as shown in table 4 .

\begin{tabular}{|l|c|c|c|}
\hline \multirow{2}{*}{} & \multicolumn{2}{|c|}{ MRT group } & \multirow{2}{*}{ p-value } \\
\cline { 2 - 3 } & \multicolumn{2}{|c}{$\overline{\mathbf{x}} \pm \mathbf{~}$} & \\
\cline { 2 - 3 } & Pre & Post & \\
\hline VAS & $6.30 \pm 0.823$ & $3.20 \pm 0.789$ & 0.002 \\
\hline Flexion & $6.30 \pm 0.675$ & $8.90 \pm 1.197$ & 0.004 \\
\hline Extension & $1.80 \pm 0.789$ & $3.80 \pm 0.919$ & 0.004 \\
\hline $\begin{array}{l}\text { Lateral flexion } \\
\text { (right) }\end{array}$ & $10.1 \pm 1.91$ & $13.7 \pm 1.56$ & $<0.005$ \\
\hline $\begin{array}{l}\text { Lateral flexion } \\
\text { (left) }\end{array}$ & $9.90 \pm 1.52$ & $13.7 \pm 1.56$ & $<0.005$ \\
\hline Rotation (right) & $4.7 \pm 0.67$ & $7.1 \pm 0.56$ & $<0.005$ \\
\hline Rotation (left) & $5.00 \pm 0.66$ & $7.2 \pm 0.78$ & $<0.005$ \\
\hline LBP index & $31.690 \pm$ & $19.460 \pm$ & \multirow{2}{*}{0.005} \\
\hline
\end{tabular}

Table 3: Mean and \pm SD, and univariate test between pre and post treatment of the dependent variables in each group.

*SD: Standard Deviation, P: Probability.

Post treatment there was a significant difference between group (A) and (B) in pain level where P-value was $(<0.05)$, and there was significant difference between group (A) and (B) in ROM where the P-value was $(<0.05)$ as shown in table 4.

\section{Discussion}

The current study revealed that the active release technique (ART) and Myofascial Release Technique (MFR) for one week in patients with low back pain resulted in significant improvement in ROM and function disability in the post-treatment, associated with a significant decrease in pain intensity in the post-treatment in both groups. Also, it revealed that there was a significant difference between Active release technique (ART) and Myofascial Release Technique (MFR) as regard to pain reduction, ROM and function disability improvement, as Active release technique allows a greater degree of pain relief and ROM and function improved than the Myofascial Release Technique.

Twenty patients with low back pain aged 20 to 50 years, were assigned into two groups, subjects in the first group (A) Active release technique (ART) (7 sessions, 1 session per day for a week). Subjects in the second group (B) received the Myofascial Release Technique (7 sessions, 1 session per day for one week). Pain sever- 


\begin{tabular}{|c|c|c|c|c|c|c|}
\hline & \multirow{2}{*}{\multicolumn{2}{|c|}{$\begin{array}{c}\text { ART group } \\
\overline{\mathbf{x}} \pm \mathbf{s} \\
\end{array}$}} & \multirow{3}{*}{ p-value } & \multirow{2}{*}{\multicolumn{2}{|c|}{$\begin{array}{c}\text { MRT group } \\
\overline{\mathbf{x}} \pm \mathbf{s} \\
\end{array}$}} & \multirow{3}{*}{ p-value } \\
\hline & & & & & & \\
\hline & Pre & Post & & pre & Post & \\
\hline VAS & $6.40 \pm 0.966$ & $1.20 \pm 0.632$ & 0.004 & $6.30 \pm 0.823$ & $3.20 \pm 0.789$ & 0.002 \\
\hline Flexion & $5.20 \pm 0.789$ & $9.20 \pm 0.632$ & 0.005 & $6.30 \pm 0.675$ & $8.90 \pm 1.197$ & 0.004 \\
\hline Extension & $1.90 \pm 0.738$ & $4.6 \pm 0.699$ & 0.004 & $1.80 \pm 0.789$ & $3.80 \pm 0.919$ & 0.004 \\
\hline $\begin{array}{l}\text { Lateral flexion } \\
\text { (right) }\end{array}$ & $9.5 \pm 2.12$ & $14.4 \pm 2.06$ & $<0.005$ & $10.1 \pm 1.91$ & $13.7 \pm 1.56$ & $<0.005$ \\
\hline $\begin{array}{l}\text { Lateral flexion } \\
\text { (left) }\end{array}$ & $9.5 \pm 2.12$ & $14.4 \pm 2.06$ & $<0.005$ & $9.90 \pm 1.52$ & $13.7 \pm 1.56$ & $<0.005$ \\
\hline Rotation (right) & $4.7 \pm 0.67$ & $8.1 \pm 0.56$ & $<0.005$ & $4.7 \pm 0.67$ & $7.1 \pm 0.56$ & $<0.005$ \\
\hline Rotation (left) & $5.00 \pm 0.66$ & $8.2 \pm 0.78$ & $<0.005$ & $5.00 \pm 0.66$ & $7.2 \pm 0.78$ & $<0.005$ \\
\hline LBP index & $31.860 \pm 12.2448$ & $14.660 \pm 4.8983$ & 0.005 & $31.690 \pm 13.1575$ & $\begin{array}{c}19.460 \pm \\
6.7421\end{array}$ & 0.005 \\
\hline
\end{tabular}

Table 4: Mean and \pm SD, and univariate test of the dependent variables for both groups.

*SD: Standard Deviation, P: Probability.

ity, ROM, and function disability were assessed before and after one week of treatment for both groups.

\section{Group of patients using active release technique}

From the statistical analysis of pre and post-treatment of pain assessment in patients receiving active release technique, there was a significant difference in pain intensity, ROM, and functional disability value between pre-treatment value and post-treatment value.

ART is a method for treating soft tissues such as tendon, nerve, and myofascial and is performed for repetitive strain injury, acute injury, and damage to functional fixation due to abnormal longterm posture. In addition, ART eliminates the adhesion of scar tissue and soft tissue causing discomfort, spasm, muscle fatigue, tingling and other symptoms [11].

Robb., et al. (2011) showed immediate muscle PPT improvements when ART was used to treat patients with an adductor strain. In addition, in a study conducted by Tak., et al. (2013), 3 weeks of ART treatment on a patient's gluteus medius with low back pain resulted in an improvement in the patient's VAS score and PPT [12].

Tak., et al. 2013 and Robb., et al. 2011 Significant improvement was observed in the VAS score, PPT and ROM following the use of
ART in the present study to treat the back muscles. It is our belief that after treatment these changes in VAS score and PPT result from reductions in muscle tone following removal of scar tissue adherent to soft tissue [13].

In a 2006 [14] study by George., et al. involving 20 young men with no lower extremity injury, hamstring flexibility increased immediately after application of ART. Similarly, in this study, ROM increased significantly after 1 week of applying ART to the back.

\section{Group of patients using myofascial release technique}

From the statistical analysis of pre and post-treatment of pain assessment in patients received myofascial release technique, there was a significant difference in pain intensity, ROM, and functional disability value between pre-treatment value and post-treatment value.

In a research Arguisuelas., et al. (2017) [15] the effectiveness of the MFR therapy sessions in 4 non-specific CLBP patients. The control group was made up of patients who had used fictional MFR. In the group shown to decrease the pain intensity (McGill Pain Questionnaire, MPQ VAS), improve efficiency in daily activity performance (pain questionnaire and Roland Morris), and reduce pain fear (questionnaire Fear-Avoidance Beliefs, FABQ). No statis- 
tically significant differences between group of study and group of controls.

In a study conducted by Seong Hun Yu., et al. (2016) [16] in women between the ages of 65 years or older complain about CLBP following application of MFR significant reduction in pain intensity (VAS), increased spine motion range (Test Schober), and improved stability body (Test stabiliometric platform).

To assess if the MFR increases the effectiveness of physiotherapy used Ajimsha., et al. (2014) [17] conducted a study to assess the efficacy of specific MFR and spine muscle exercise group nurses CLBP. The control group consisted of patients who were treated with the same exercise. Treatment lasted 8 weeks ( 24 therapy sessions). The assessment of efficacy was carried out immediately after and after 8 and 12 weeks. The results showed significantly better results in terms of pain reduction (MPQ) and improved functional capacity (Quebec Back Pain Disability Scale) in the group that used the MFR. In the group in which the MFR was used as a reduction of pain by $53.3 \%$ (in the group, which used classes - 26.1\%) and $30 \%$ improvement in functional capacity (in the group, which used classes - $9.8 \%$.

Paul J., et al. (2017) [18] who compared the effect of MFR and deep transverse friction massage for upper trapezius trigger point, explained that MFR improves vertical alignment and lengthens the body providing more room for proper functioning of osseous structures, nerves, muscles, blood vessels and organs that enhance function.

\section{Comparison between groups after treatment}

Comparing the effect of active release technique and myofascial release technique on low back pain. After treatment, there was a significant difference between active release technique and myofascial release technique as regard to pain reduction, ROM and function improvement as active release technique allows a greater degree of pain relief and greater improvement in ROM and function than the myofascial release technique for patients with low back pain.

Mishra D., et al. (2018) [19] comparison of active release technique and myofascial release technique Upper Trapezius Spasm reported that both techniques are effective in relieving symptoms and related disability in upper trapezius muscle spasm, Active Re- lease Technique gave better results compared with Myofascial Release Technique.

\section{Limitations of the Study}

Among the limitations of the study were small sample size, Individual variations, and possible errors during measurement procedures.

\section{Conclusion}

There was a significant difference between active release technique and myofascial release technique as regard to pain reduction, improvement ROM and functional disability. Which active release technique allows a greater degree of pain relief and allows a greater improvement in ROM and function disability than the myofascial release technique for patients with low back pain.

\section{Conflict of Interest}

None declared.

\section{Bibliography}

1. Kopec JA., et al. "Predictors of back pain in a general population cohort". Spine 29.1 (2004): 70-77.

2. Méndez FJ and Gómez-Conesa A. "Postural hygiene program to prevent low back pain”. Spine 26.11 (2001): 1280-1286.'

3. Filiz M., et al. "The effectiveness of exercise programmes after lumbar disc surgery: a randomized controlled study". Clinical Rehabilitation 19.1 (2005): 4-11.

4. Werneke M and Hart DL. "Centralization phenomenon as a prognostic factor for chronic low back pain and disability". Spine 26.7 (2001): 758-764.

5. Magee DJ., et al. "Pathology and intervention in musculoskeletal rehabilitation Elsevier Health Sciences 3 (2015).

6. Howitt SD. "Lateral epicondylosis: a case study of conservative care utilizing ART® and rehabilitation". The Journal of the Canadian Chiropractic Association 50.3 (2006): 182.

7. Simons DG and Travell JG. "Myofascial origins of low back pain: 3. Pelvic and lower extremity muscles". Postgraduate Medicine 73.2 (1983): 99-108. 
8. Bijur PE., et al. "Reliability of the visual analog scale for measurement of acute pain". Academic Emergency Medicine 8.12 (2001): 1153-1157.

9. Reese NB and Bandy WD. "Joint range of motion and muscle length testing-E-book. Elsevier Health Sciences (2016).'

10. Fairbank JC and Pynsent PB. "The Oswestry disability index". Spine 25.22 (2000): 2940-2953.

11. Brian A., et al. "Release Your Pain: Resolving Repetitive Strain Injuries with Active Release Techniques". Pub Group West (2005): 15-29.

12. Robb A and Pajaczkowski J. "Immediate effect on pain thresholds using active release technique on adductor strains: pilot study". Journal of Bodywork and Movement Therapies 15.1 (2011): 57-62.

13. Tak S., et al. "The effects of active release technique on the gluteus medius for pain relief in persons with chronic low back pain". Physical Therapy Rehabilitation Science 2.1 (2013): 2730.

14. George JW., et al. "The effects of active release technique on hamstring flexibility: a pilot study". Journal of Manipulative and Physiological Therapeutics 29.3 (2006): 224-227.

15. Arguisuelas MD., et al. "Effects of myofascial release in nonspecific chronic low back pain: a randomized clinical trial”. Spine 42.9 (2017): 627-634.

16. Yu SH., et al. "The effect of abdominal drawing-in exercise and myofascial release on pain, flexibility, and balance of elderly females". Journal of Physical Therapy Science 28.10 (2016): 28122815.

17. Ajimsha MS., et al. "Effectiveness of myofascial release in the management of chronic low back pain in nursing professionals". Journal of Bodywork and Movement Therapies 18.2 (2014): 273-281.

18. Paul J and Kumar M. "A comparative study on the effect of Myofascial Release versus Deep Transverse Friction on myofascial trigger points of upper back". International Journal of Medical 2 (2013): 27-30.
19. Mishra D., et al. "Comparative Study of Active Release Technique and Myofascial Release Technique in Treatment of Patients with Upper Trapezius Spasm". Journal of Clinical and Diagnostic Research 12.11 (2018).

\section{Volume 4 Issue 8 August 2021 \\ (C) All rights are reserved by Hamza M Shaheen., et al.}

